University of Nebraska - Lincoln

DigitalCommons@University of Nebraska - Lincoln

November 2000

\title{
Cognitive consequences and central nervous system injury following treatment for childhood leukemia
}

I. M. Moore

K. A. Espy

University of Nebraska-Lincoln, kespy2@unl.edu

P. Kaufmann

J. Kramer

K. Kaemingk

See next page for additional authors

Follow this and additional works at: https://digitalcommons.unl.edu/dcnlfacpub

Part of the Neurosciences Commons

Moore, I. M.; Espy, K. A.; Kaufmann, P.; Kramer, J.; Kaemingk, K.; Miketova, P.; Mollova, N.; Kaspar, M.; Pasvogel, A.; Schram, K.; Wara, W.; Hutter, J.; and Matthay, K., "Cognitive consequences and central nervous system injury following treatment for childhood leukemia" (2000). Developmental Cognitive Neuroscience Laboratory - Faculty and Staff Publications. 21.

https://digitalcommons.unl.edu/dcnlfacpub/21

This Article is brought to you for free and open access by the Developmental Cognitive Neuroscience Laboratory at DigitalCommons@University of Nebraska - Lincoln. It has been accepted for inclusion in Developmental Cognitive Neuroscience Laboratory - Faculty and Staff Publications by an authorized administrator of DigitalCommons@University of Nebraska - Lincoln. 


\section{Authors}

I. M. Moore, K. A. Espy, P. Kaufmann, J. Kramer, K. Kaemingk, P. Miketova, N. Mollova, M. Kaspar, A.

Pasvogel, K. Schram, W. Wara, J. Hutter, and K. Matthay 


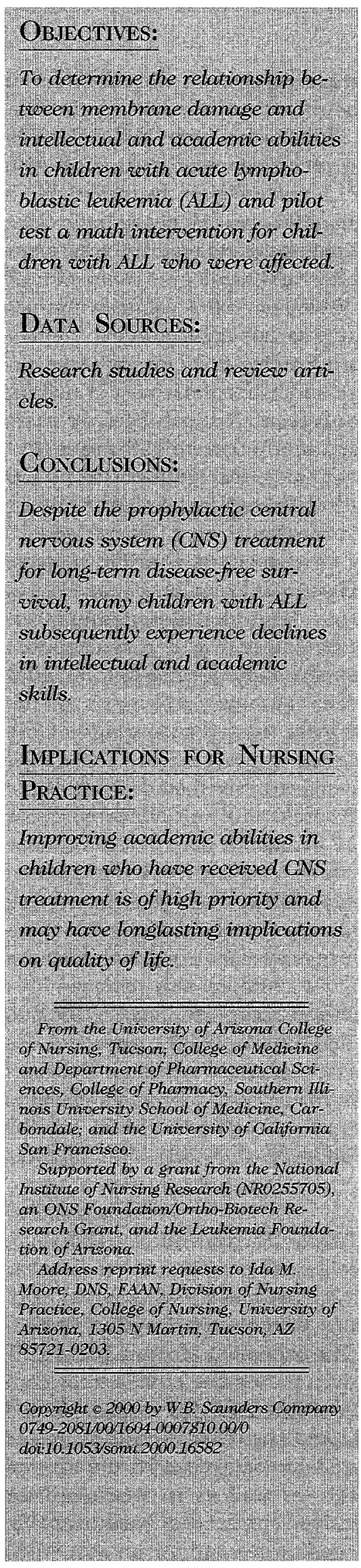

\title{
Cognitive
}

Consequences AND Central Nervous SYSTEM INJURY Following Treatment FOR CHILDHOOD LEUKEMIA

\author{
IDA M. MOORE, KIMBERLY ANDREWS ESPY, \\ PaUl KaUfmann, Joel Kramer, Kris KaemingK, \\ Petra Miketova, Nevena Mollova, Marta Kaspar, \\ Alice Pasvogel, Karl Schram, William Wara, \\ JohN HutTER, and Kate MatThaY
}

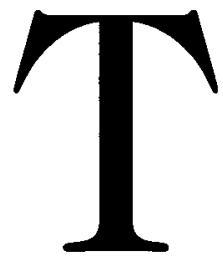

HE 5-YEAR relative survival rate from acute lymphoblastic leukemia (ALL) for children under 15 years of age is approximately $80 \% .^{1,2}$ The routine use of central nervous system (CNS) therapy contributed to the progress in long-term disease-free survival by significantly reducing the incidence of meningeal leukemia from $75 \%$ or $80 \%$ to less than $10 \% .^{3}$ Whole brain radiation, intrathecal chemotherapy, and high-dose systemic chemotherapy are effective modalities for reducing the risk of meningeal relapse and are therefore used as prophylactic GNS treatment. ${ }^{4}$

Despite the importance of prophylactic GNS treatment for longterm disease-free survival, many children with ALL subsequently experience declines in intellectual and academic skills. Whole brain radiation has been most closely linked to CNS late effects ${ }^{5-21}$; however, studies have shown that intrathecal chemotherapy and high-dose systemic chemotherapy also are associated with specific intellectual and academic deficits. ${ }^{10,22-26}$ Declines in intellectual and academic function are thought to be due to treatment-related effects on nonmalignant cells in the CNS; however, the specific mechanisms are not well understood. A variety of neuroimaging and electrophysiologic methods have been used to investigate white matter changes, cerebral calcifications and atrophy, perfu- 
sion defects, and abnormal glucose metabolism in children with ALL who were undergoing or who had completed CNS treatment with whole brain radiation and/or intrathecal chemotherapy. ${ }^{27-36}$ Three of the five studies that included measures of intellectual and academic function demonstrated a relationship between CNS changes and functional decline..$^{29,31,32}$

The purpose of the first part of this research program (referred to subsequently as study 1 ) was to investigate membrane damage to nonmalignant cells in the CNS and to determine the relationship between membrane damage and intellectual and academic abilities. The purpose of the second component of the research program (study 2) was to pilot test a math intervention for children with ALL who had documented declines in their academic and arithmetic abilities.

\section{Study 1: Gell Membrane Damage and}

\section{Intellectual AND Academic Abilities}

$\mathbf{R}$ adiation and chemotherapy damage cell membranes by activation of phospholipase $\mathrm{A}_{2} \cdot{ }^{37}$ Membrane damage may be an initial event that precedes more obvious CNS changes, such as loss of white matter, decreased perfusion, and cerebral atrophy. Changes in cerebrospinal fluid (CSF) phospholipid concentration reflect degradation of the lipid bilayer of cell membranes ${ }^{38}$ and may be useful as a marker of early CNS damage and later declines in intellectual and academic skills. Furthermore, GSF phospholipid composition is believed to reflect the exchange of lipids between the lipoproteins of the CSF and the surrounding brain tissue. The specific phospholipids of interest were phosphatidylcholine (PC) and sphingomyelin (SM) since they account for approximately $60 \%$ and $24 \%$, respectively, of total CSF phospholipids. ${ }^{39}$ It was hypothesized that increased CSF PC + SM during ALL CNS treatment would be negatively correlated with future intellectual and academic abilities.

\section{Materials and Methods}

Subjects. Children with newly diagnosed ALL were recruited from two childhood cancer treatment centers. Consent was obtained from parents or legal guardians and from children aged 7 years or older. The total sample comprised 41 children. Nine of the 41 children received whole brain radiation in combination with intrathecal chemo- therapy. This article reports on the 32 children who received only intrathecal chemotherapy. Seventeen subjects (53\%) were male and $15(47 \%)$ were female; the mean age at the time of ALL diagnosis was $73.11 \pm 46.79$ months (range, 20 to 206 months). One child was diagnosed before the age of 2 years. Sixteen subjects received treatment according to Pediatric Oncology Group (POG) protocols 8602,9005 , or 9006 . Central nervous system treatment was triple intrathecal chemotherapy with methotrexate $(12 \mathrm{mg})$, cytosine arabinoside (12 $\mathrm{mg})$, and hydrocortisone ( $24 \mathrm{mg}$ ) for children aged 2 to 9 years. Subjects aged 10 years and older received methotrexate $(15 \mathrm{mg})$, cytosine arabinoside (30 $\mathrm{mg})$, and hydrocortisone (30 $\mathrm{mg})$. Intrathecal drug doses for the child who was younger than 2 years were $10 \mathrm{mg}, 10 \mathrm{mg}$, and 20 $\mathrm{mg}$, respectively. Fourteen of the 16 subjects treated with POG protocol regimens received six $(n=11)$ or $12(n=3)$ cycles of systemic intermediate-dose methotrexate $\left(1 \mathrm{~g} / \mathrm{m}^{2}\right)$; two children received 12 cycles of oral methotrexate $\left(30 \mathrm{mg} / \mathrm{m}^{2}\right.$ every 6 hours over 36 hours). Thirteen of the other 16 subjects were treated according to Children's Cancer Group protocols 1881, 1891, or 1922. They received intrathecal methotrexate (12 $\mathrm{mg}$ ) every 1 to 2 weeks during CNS prophylaxis and then every 12 weeks for 2 to 3 years. Three subjects were treated according to Children's Cancer Group protocol 1901, receiving intrathecal methotrexate $(12 \mathrm{mg})$ weekly during consolidation and then every 8 weeks during maintenance therapy. These three children also received lowdose systemic methotrexate $\left(200 \mathrm{mg} / \mathrm{m}^{2}\right)$ for 2 to 3 years. There was no significant difference in maternal education between the triple intrathecal chemotherapy group $(13.93 \pm 2.02$ years $)$ and the intrathecal methotrexate group $(14.53 \pm 2.9$ years; $\mathrm{t}=-.73, \mathrm{df}=27, P=.82$ ).

\section{Procedures}

Cerebrospinal fluid phospholipids. One milliliter CSF was collected during routine lumbar punctures for GNS treatment and evaluation of GNS disease status during week 1 and week 6 of ALL treatment. The collection of subsequent CSF samples was determined by the child's treatment regimen. The remaining samples were collected between weeks 12 and 16 , weeks 20 and 25 , weeks 40 and 44 , weeks 52 and 56, weeks 78 and 82 , and weeks 96 and 110 , as well as in week 140 for children who received intrathecal methotrexate. Samples were placed on ice and frozen at $-80^{\circ} \mathrm{C}$. 
Phospholipids were extracted from CSF samples according to the method reported by Folch et al. ${ }^{40}$ Phospholipid standards (Sigma, St. Louis) and CSF lipid extracts were separated on a model 338 Gold HPLC system (Beckman, San Ramon, CA) and a prepacked silica column based on the method reported by Chen and Chan. ${ }^{41}$ High-performance liquid chromatography fractions from patient samples were collected for confirmatory analysis by fast atom bombardment mass spectrometry on a custom-built four-sector instrument of BEBE geometry (AMD Intrectra, Harpstedt, Germany). Structural confirmation of $\mathrm{PC}+\mathrm{SM}$ by fast atom bombardment mass spectrometry has been previously reported ${ }^{38}$ as well as coelution of some SM molecular species in the PG fraction. ${ }^{38}$

Intellectual and acodemic abilities. Intellectual abilities were assessed using the Wechsler intelligence scale for children revised (WISC-R) ${ }^{42}$ for children aged 6 years and older and the McGarthy scales of children's abilities (MSCA) ${ }^{43}$ for children aged 5 years or younger. Relevant indices from each test were combined to measure abilities across the age span of subjects. The WISC-R full scale IQ score and the MSCA general intellectual index were used to measure overall intellectual abilities (IQ). Verbal intellectual skills were measured with the WISC-R verbal IQ and the MSCA verbal scale index, whereas the WISG-R performance IQ and the MSCA perceptual performance scale index assessed nonverbal or performance-based intellectual skills.

Academic achievement of children aged 5 years and older was evaluated with the wide range achievement test-revised (WRAT-R) ${ }^{44}$ Standard scores from the reading, spelling, and arithmetic subtests were used. The MSCA number questions subtest was used to assess emergent arithmetic skills in children younger than 5 years; however, pre-emergent reading and spelling skills could not be estimated.

A pediatric neuropsychologist conducted the assessment of intellectual and academic abilities. The early assessment was completed approximately 7 months after the diagnosis of ALL (mean, $7.22 \pm 4.32$ months) to minimize the influence of fatigue, emotional distress, and hospitalizations on intellectual and academic performance. The late evaluation was conducted approximately 52 months after the diagnosis of ALL (mean, $52.85 \pm$ 8.39 months).

Data analysis. Descriptive statistics were computed on the demographics, intellectual and academic abilities, and CSF SM and PC + SM variables. The mean CSF $\mathrm{SM}$ and $\mathrm{PC}+\mathrm{SM}$ values at week 1 were compared ( $t$-test for matched pairs) with mean values at subsequent weeks to examine significant changes during the course of ALL. Because of coelution of some SM molecular species in the PC fraction, the concentration of the combined PG + SM fraction and the SM fraction alone were correlated with intellectual and academic abilities. Intellectual and academic scores from the early evaluation were correlated with the increase in CSF SM and the combined $\mathrm{CSF}$ PC + SM above baseline values at weeks 6 and 12 to 16 . Scores from the late evaluation were correlated with the increase in SM and PC + SM above baseline levels at weeks 12 to 16 and weeks 40 to 44 . Although the increase in CSF phospholipid concentrations at weeks 96 to 104 were elevated significantly in children receiving intrathecal methotrexate only, the phospholipids at this time interval were correlated with late intellectual and academic abilities to determine the relationship of late GSF changes with outcome. The two children in the triple intrathecal chemotherapy group who received 12 cycles of oral methotrexate instead of intermediate-dose systemic methotrexate were excluded from the correlational analyses.

\section{Results}

The mean CSF PC $+\mathrm{SM}$ at week 1 was 1.19 $\mu \mathrm{g} / \mathrm{mL}$ for the triple intrathecal chemotherapy group and $1.21 \mu \mathrm{g} / \mathrm{mL}$ for the intrathecal methotrexate group. The concentration of the $\mathrm{PC}+\mathrm{SM}$ fraction increased in both groups from week 1 through weeks 96 to 110 , with significant increases at weeks 12 to 16 and weeks 40 to 44 for both groups. Additionally, $\mathrm{PC}+\mathrm{SM}$ increased significantly at weeks 52 to 56 in the triple intrathecal chemotherapy group and at week 6 and weeks 20 to 25 in the intrathecal methotrexate group.

The mean CSF SM was $0.24 \mu \mathrm{g} / \mathrm{mL}$ and 0.28 $\mu \mathrm{g} / \mathrm{mL}$ for the triple intrathecal chemotherapy and intrathecal methotrexate groups, respectively, at week 1; it was increased at all subsequent measurement intervals. Statistically significant increases were observed at week 6 , weeks 12 to 16 , weeks 20 to 25 , and weeks 96 to 110 in the intrathecal methotrexate group and at weeks 40 to 44 in the triple intrathecal chemotherapy group. Table 1 summarizes the CSF SM and PC + SM by 


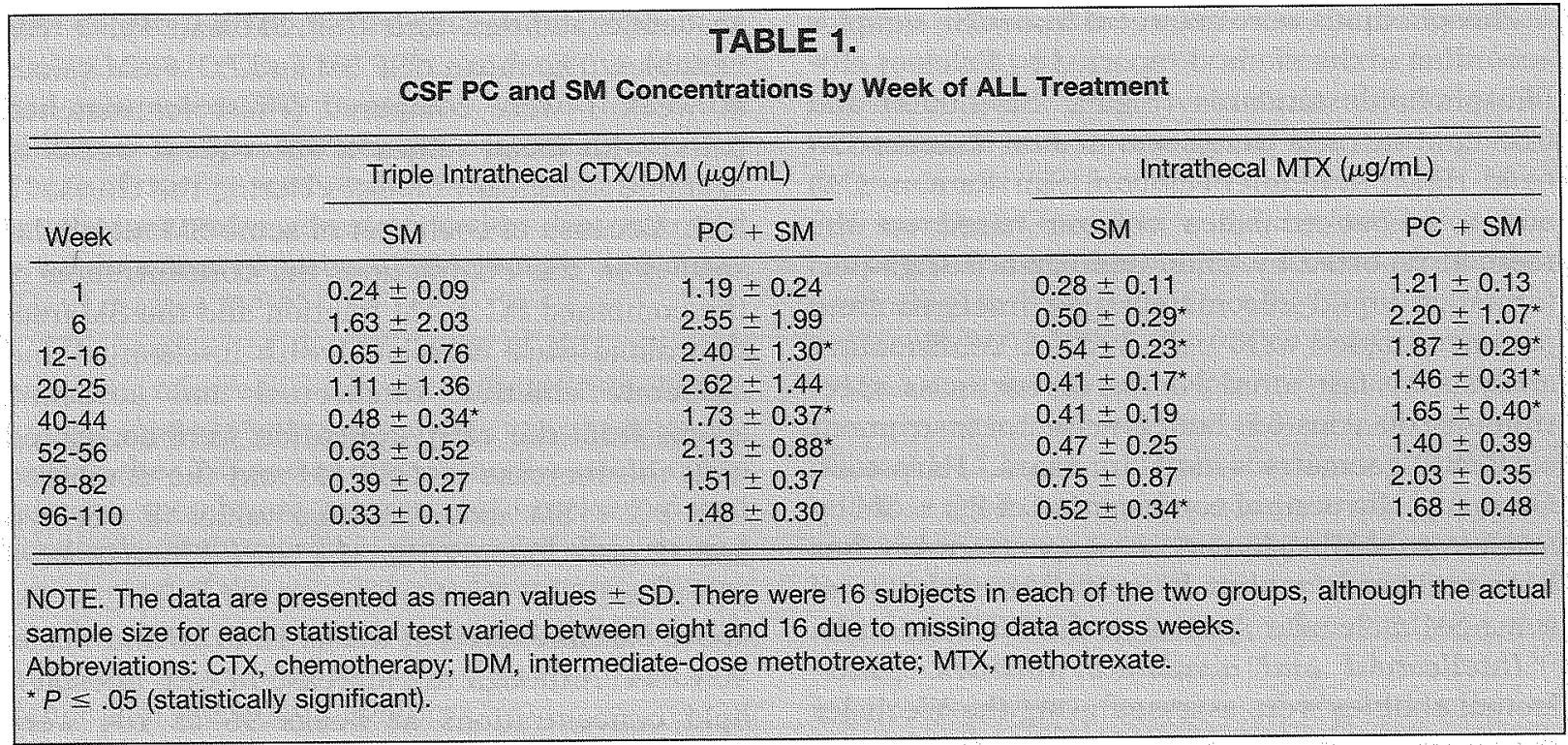

week of ALL treatment and by CNS treatment group.

The mean overall intelligence, verbal intelligence, and nonverbal intelligence were within the average to above-average range at both evaluations. Mean overall and verbal intelligence scores were significantly higher in the intrathecal methotrexate group than in the triple intrathecal chemotherapy group at the baseline evaluation $(P \leq$ $.05)$ but not at the late evaluation. Overall intellectual and performance abilities increased in the triple intrathecal chemotherapy group from the early to late evaluation $(P=.05)$.

The reading, spelling (for children aged 5 years and older), and arithmetic mean scores were also within the average range at the baseline evaluation, and there were no significant differences between the two groups. Performance on arithmetic declined an average of 9 standard score points in the intrathecal methotrexate group $(P=$ $.05)$ and 10 standard score points in the triple intrathecal chemotherapy group $(P=.01)$. Table 2 summarizes the results of the intellectual and academic abilities for the two CNS treatment groups.

There were no significant negative correlations between week 1 CSF phospholipids and baseline scores on measures of intellectual and academic abilities. The increase over baseline levels of CSF $\mathrm{SM}$ and $\mathrm{PC}+\mathrm{SM}$ at week 6 and weeks 12 to 16 were negatively correlated with early intellectual and academic arithmetic in the triple intrathecal chemotherapy group $(r=-.11$ to -.78$)$. Most correlations between early intellectual abilities were also negatively correlated with SM or $\mathrm{PG}+$ $\mathrm{SM}$ in the intrathecal methotrexate group; however, the correlations were weaker than those in the triple intrathecal chemotherapy group $(r=$ -.03 to -.50 ). Ten of the 11 significant correlations between GSF phospholipids and early intellectual and academic achievement scores were found in the triple intrathecal group (Table 3).

The early increase in CSF SM and PG + SM were negatively correlated with late intellectual and academic abilities in the triple intrathecal chemotherapy group $(r=-.41$ to -.84$)$. However, with the exception of academic arithmetic, the early increase in phospholipids was not strongly correlated with intellectual and academic abilities in the intrathecal methotrexate group. Eight of the nine significant correlations between early increases in CSF phospholipids and late abilities were found in the triple intrathecal chemotherapy group.

The opposite pattern was found with the late increase in phospholipids. The late increase in SM was negatively correlated with all measures of intellectual and academic abilities in the intrathecal methotrexate group $(r=-.69$ to -.89$)$, but only with academic arithmetic in the triple intratheeal chemotherapy group. Eight of the nine significant correlations occurred in the intrathecal methotrexate group. Table 4 summarizes the correlations between CSF phospholipid concentra- 


\begin{tabular}{|c|c|c|}
\hline \multicolumn{3}{|c|}{$\begin{array}{l}\text { Intellectual and Academic Abilities } \\
\text { by CNS Treatment Group }\end{array}$} \\
\hline Ability & Early & Late \\
\hline \multicolumn{3}{|l|}{ Intelectual overall } \\
\hline $\begin{array}{l}\text { Triple intrathecal } \\
\text { CTXIDM }\end{array}$ & $97.8 \pm 11.2^{x}$ & $102.2+13.71$ \\
\hline Intrathecal MTX & $112.5 \div 13.0^{x}$ & $112.5 \div 15.9$ \\
\hline Verbal & & \\
\hline $\begin{array}{l}\text { Triple intrathecal } \\
\text { CTXIDM }\end{array}$ & $95.5+13.9$ & $96.9+14.1 x$ \\
\hline Intrathecal MTX & $115.5+14.6^{\circ}$ & $114.0=14.1 \%$ \\
\hline Performance & & \\
\hline $\begin{array}{l}\text { Triple intrathecal } \\
\text { CTXIDM }\end{array}$ & $9.5 \pm 13.9^{\circ}$ & $102.2+13.71$ \\
\hline Intrathecal MTX & $115.5 \pm 14.6$ & $108.4 \div 17.3$ \\
\hline Academic reading & & \\
\hline $\begin{array}{l}\text { Triple intrathecal } \\
\text { CTXubM }\end{array}$ & NA & $92.3=14.2$ \\
\hline Intrathecal NTX & NA & $95.5+167$ \\
\hline Academic spelling & & \\
\hline $\begin{array}{l}\text { Triple intrathecal } \\
\text { otxibu }\end{array}$ & NA & $93.3=18.4$ \\
\hline Intrathecal MTX & NA & $94.1+12.8$ \\
\hline Academic arithmetic & & \\
\hline $\begin{array}{l}\text { Triple intrathecal } \\
\text { CTXIDM }\end{array}$ & $99.0+20.2$ & $89.0-15.6 t$ \\
\hline Intrathecal NTX & $104.4=13.0$ & $95.5 \div 16.5 t$ \\
\hline
\end{tabular}

NOTE. The data are presented as mean values $\pm \mathrm{SD}$. There were 16 subjects in each of the two groups, although the actual sample size for each statistical test varied between 10 and 16.

Abbreviations: NA, not available (mean early scores not reported because of number of subjects younger than 5 years at time of evaluation), CTX, chemotherapy, IDM, intermediate dose methotrexate; NTX, methotrexate.

* Triple Intrathecal CTXIDM and Intrathecal MTX group mean scores significantly different $(P=05)$.

t Mean early and late evaluation scores significantly different $(P \leq .05)$.

tions and the decline in intellectual and academic abilities at the late evaluation.

\section{Discussion}

To our knowledge, there are no previously reported studies of changes in CSF phospholipid concentration in children with ALL receiving intratheeal methotrexate or triple intrathecal chemotherapy. Alterations of membrane phospholipids have been previously related to inflammation, edema, and ischemia in the CNS. ${ }^{45,46} \mathrm{~A}$ significant increase in the CSF phospholipids was observed during the first 3 months of ALL treatment that persisted through the first year of ALL treatment.
This finding suggests that increased concentrations of CSF phospholipids may be a measure of the initial or acute phase in the pathogenesis of CNS injury. The CSF samples analyzed in this study were collected 1 to several weeks after the previous intrathecal chemotherapy or systemic intermediate-dose methotrexate treatment. Therefore, it is likely that our data do not represent the maximum increase in phospholipid concentration in the CSF.

Fourteen of the 16 children treated on POG protocols received systemic intermediate-dose methotrexate $\left(1 \mathrm{~g} / \mathrm{m}^{2}\right)$ during the first year of ALL treatment. Triple intrathecal chemotherapy was coadministered during systemic therapy. Balis and Poplack ${ }^{4}$ report that CSF (methotrexate) of 0.2 to $0.3 \mu \mathrm{m}$ are achieved after systemic intermediate-dose methotrexate, but coadministration of intrathecal methotrexate raises the CSF (methotrexate) to over $1 \mu \mathrm{m} .{ }^{4}$ Interestingly, baseline intellectual and academic skills were related to early CSF phospholipid concentrations primarily in the triple intrathecal chemotherapy group who received dual systemic intermediate-dose methotrexate and triple intrathecal therapies before the baseline evaluation. Therefore, the administration of dual therapies early in treatment may result in earlier neurotoxicity in the triple intrathecal group, which is primarily manifested in the early assessment scores.

The concentration of CSF phospholipids during the first year of ALL treatment was greater in children who received triple intrathecal chemotherapy, relative to those who received intrathecal methotrexate. Therefore, membrane damage may occur earlier in the triple intrathecal chemotherapy group as evidenced by the significant negative correlations with intellectual and academic abilities at the early evaluation. Improvement in IQ scores may reflect some level of recovery and/or a practice effect.

The administration of triple intrathecal chemotherapy with doses of systemic methotrexate of 1 $g / \mathrm{m}^{2}$ or greater has been associated with grades 3 and 4 neurotoxicity. ${ }^{47}$ The most common symptoms include generalized and focal neurologic seizures; two thirds of all seizures occurred after completion of intensification with systemic intermediate-dose methotrexate. The investigators concluded that repetitive doses of methotrexate at $1 \mathrm{~g} / \mathrm{m}^{2}$ may double the risk for neurotoxicity in comparison to low-dose methotrexate when combined with triple intrathecal chemotherapy pro- 


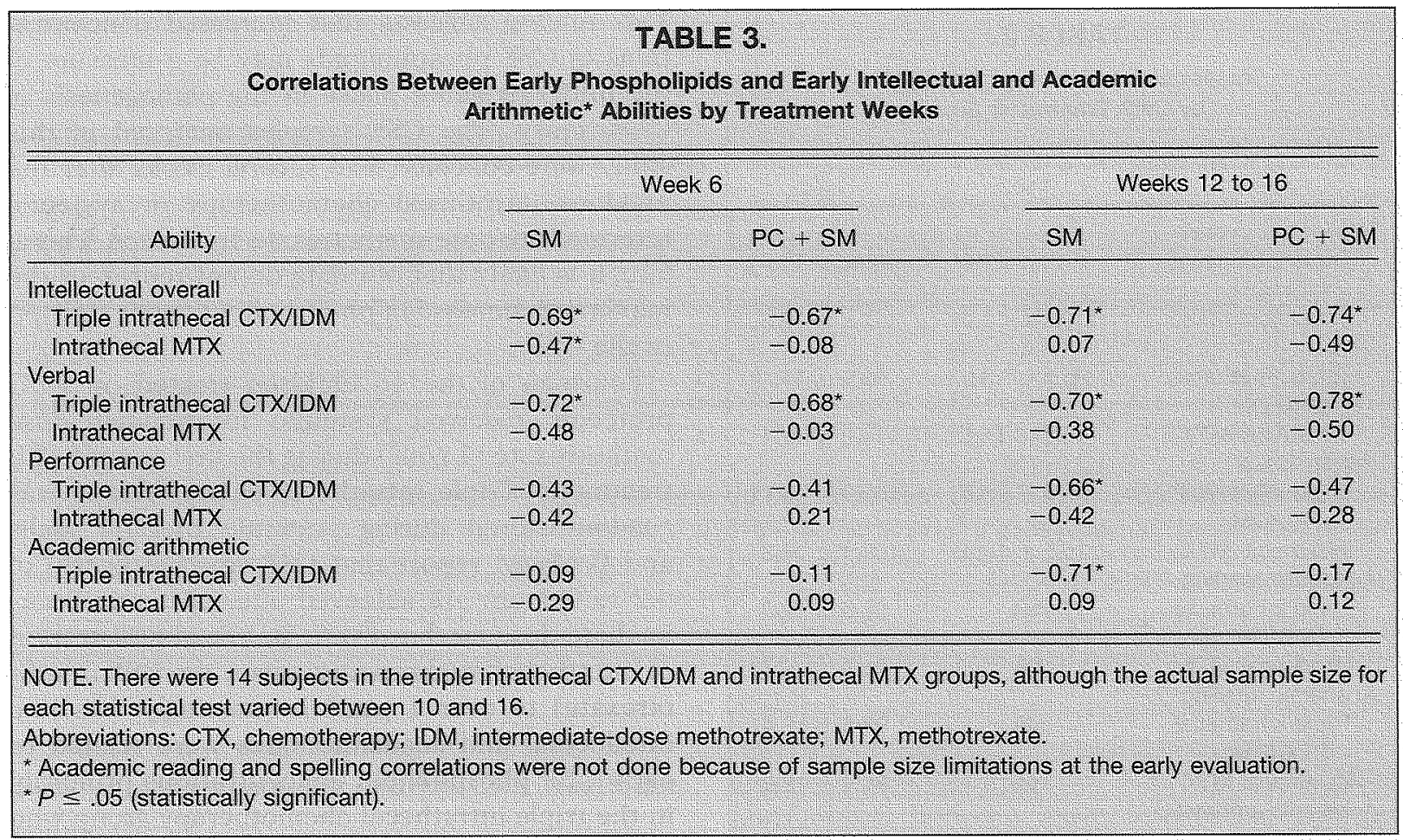

phylaxis. It was not possible to determine the differential effects of triple intrathecal methotrexate and systemic intermediate-dose methotrexate therapy in this study because 14 of the 16 children treated on POG protocols received both.

Differences in late intellectual and academic scores among children were related to CSF levels of phospholipid concentrations in the final GSF sample primarily in the intrathecal methotrexate group. It may be that neurotoxicity is slower to accumulate with this regimen and the outcome effects are not manifested until after therapy has ended.

The decline in academic achievement in both groups raises the possibility that subtle neurologic sequelae are associated with CNS treatment regimens that do not include radiation. Performance on tests of intelligence and academic achievement requires the integration of diverse skills, from attention and perception to judgment and planning. Tests of intelligence were sensitive to changes in CSF phospholipid concentrations in children who received either chemotherapeutic approach. Because multiple discrete neuropsychological skills determine intellectual performance, these findings may represent markers for specific areas of cognitive dysfunction.

The neurotoxic risks of intensive chemother- apy, however, must be balanced with the antileukemic efficacy of the regimen. Mahoney et $1^{49}$ reported findings from POG 9005 . Early intensive therapy with 12 courses of intravenous methotrexate ( $1 \mathrm{~g} / \mathrm{m}^{2}$ over 24 hours) followed by intravenous mercaptopurine ( $1 \mathrm{~g} / \mathrm{m}^{2}$ over 6 hours) was more effective than low-dose methotrexate (30 $\mathrm{mg} / \mathrm{m}^{2}$ orally every 6 hours times six doses) followed by intravenous mercaptopurine for prevention of relapse in B-precursor lower-risk ALL. 48

Atrophy, leukoencephalopathy, calcifications, white matter change, and perfusion defects have been described in children with ALL who were undergoing or who had completed CNS treatment. ${ }^{28,29,30,33}$ Across studies, the incidence of morphologic changes was approximately $50 \%$. Hertzberg et al 29 reported a relationship between structural CNS changes and lower scores on WISC-R subtests. However, other investigators have failed to document significant correlations between neuroimaging findings and intellectual or academic abilities. $28,30,33$ Slower reaction times and longer latency intervals, as measured by event-related potential, have been reported among long-term survivors of childhood ALL, 31,34 Moderate negative correlations between the electrophysiologic measures and neuropsychological test scores (full scale IQ, performance IQ, aca- 


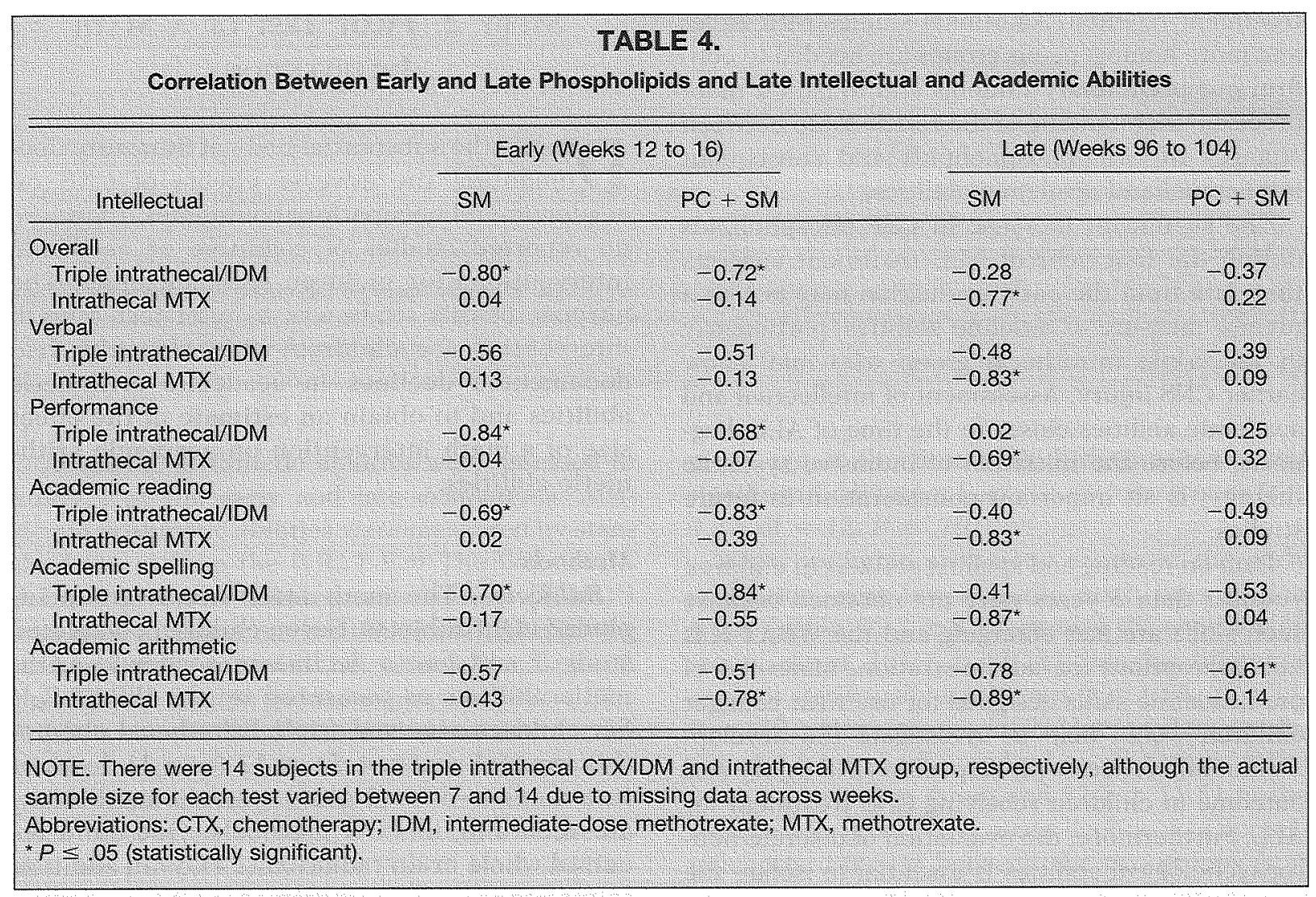

demic achievement, visuospatial, somatosensory, motor, attention, and distractibility) support Moore et al's ${ }^{31}$ hypothesis that treatment regimens that include intense CNS treatment are associated with intellectual sequelae and slowing of cortical processing.

Several recent studies have investigated other CSF markers of neurotoxicity associated with methotrexate. Surtees et $a^{49}$ reported decreased concentrations of S-adenosylmethionine and increased concentrations of myelin basic protein during presymptomatic GNS and late-intensification periods of treatment. S-adenosylmethionine is a universal methyl-donor critical for the singlecarbon transfer pathway and important for maintenance of myelin. Findings were consistent with the hypothesis that methotrexate causes CNS folate deficiency, which ultimately causes subelinical demyelination.

Methotrexate also has been shown to signifcantly increase the concentration of homocysteine and excitatory amino acids in CSF $50 \mathrm{~Pa}$ tients with neurologic toxicity at the time of CSF sample collection had some of the highest concen- trations of homocysteine and excitatory amino acids. Homocysteine is toxic to vascular endothelium and promotes thrombosis formation, while overexpression of excitatory amino acids may cause neuronal injury and death. ${ }^{51}$ These findings suggest that vascular and neuronal injury also may be involved in the pathogenesis of CNS toxicity.

\section{Limitations}

This study is an initial attempt to measure changes in CSF phospholipids during ALL treatment and to correlate the concentration of CSF phospholipids with intellectual and academic abilities. Therefore, our findings are preliminary and warrant replication. The sample comprised children treated on several different Children's Cancer Group or POG ALL protocols. Variability in the CNS treatment regimen and in the timing of CSF sample collection is a limitation. Differences in correlations between CSF phospholipids, chemotherapy protocols, and intellectual and academic abilities suggest that the optimal time for measuring GNS injury may vary by the specific 
treatment regimen. Additional studies with larger and more homogeneous groups are needed to confirm our preliminary findings and to gain a more precise understanding of the relationship between changes in CSF phospholipids and subsequent intellectual and academic abilities.

The significant increase in CSF phospholipids during the first year of ALL treatment suggests that data from the early evaluation may not be a precise measure of baseline abilities, particularly in the triple intrathecal group who may show earlier CNS injury. Assessment of intellectual and academic abilities closer to the time of ALL diagnosis, before the initiation of intensive systemic therapy, is an important consideration in future studies.

Data on reading and spelling abilities in children younger than 5 years were not obtained because such skills are just emerging and the WRAT-R is not appropriate for administration. Measures of pre-academic skills designed for use with younger children would help to investigate the developmental differences with respect to CNS injury and outcome in children receiving CNS treatment for ALL. Furthermore, the evaluation of discrete neuropsychological abilities is essential to isolate the specific areas of intellectual decline that may be related to CNS injury and CSF phospholipids.

\section{Conchusion}

In summary, the results from this study provide evidence that changes in CSF phospholipids may be related to differences in intellectual and academic outcomes in children with ALL receiving CNS treatment Increased $\mathrm{SM}$ and $\mathrm{PG}+\mathrm{SM}$ in CSF samples were observed in children who were receiving CNS treatment. The increased concentration of these phospholipids was negatively correlated with some areas of intellectual and academic skills early in the course of treatment, especially for children who were being treated with regimens that included triple intrathecal therapy, and later for those who received intrathecal methotrexate. Therefore, membrane injury, as evidenced by an increase in CSF phospholipid concentrations, is thought to reflect acute injury. The long-term effects of membrane injury on the CNS are unknown. A greater understanding of the biological meehanisms associated with CNS injury is essential to the development of pharmacologic and behavioral interventions designed to minimize the adverse effects of CNS treatment.

\section{Study 2: Pllot Test of a Math INTERVENTION}

D espite the substantial body of literature documenting the adverse effects of CNS on academic and occupational outcomes, there are no reported studies of cognitive or academic interventions for children with leukemia. The purpose of this study was to pilot test a math intervention for children with ALL who had documented declines in academic arithmetic abilities and to obtain an estimate of the effect size of a math intervention on academic arithmetic abilities.

\section{Methods}

Subjects. The math intervention group included eight subjects. Seven children completed study 1 and had a decline in academic arithmetic abilities as measured by the WRAT-R. ${ }^{4}$ Six children received triple intrathecal chemotherapy and six $(n=5)$ or $12(n=1)$ doses of intermediate-dose $\left(1 \mathrm{~g} / \mathrm{m}^{2}\right)$ systemic methotrexate according to POG protocols. One child received whole brain radiation ( $24 \mathrm{~Gy}$ ) in addition to intrathecal chemotherapy for CNS leukemia. The subject who did not participate in study 1 received intrathecal and systemic chemotherapy.

A no-intervention comparison group was included to determine whether children with ALL who did not receive the intervention also had improvements in math achievement scores. This group comprised seven subjects who also completed study 1 . All seven subjects received triple intrathecal chemotherapy. Five subjects received either $6(\mathrm{n}=4)$ or $12(\mathrm{n}=1)$ doses of intermediate-dose $\left(1 \mathrm{~g} / \mathrm{m}^{2}\right)$ systemic methotrexate. Two subjects received 12 cycles of oral methotrexate $\left(30 \mathrm{mg} / \mathrm{m}^{2}\right.$ ).

Design. A two-group longitudinal nonexperimental design was used. The math intervention provided 40 to 50 hours of a skill-based approach to teach math concepts. The specific concepts and skills were individualized according to each child's abilities and academic placement. Information on time devoted to specific concepts and skills was recorded after each session. The no-intervention comparison group was recruited for the final evaluation at a time consistent with the postintervention evaluation in the math intervention group. 


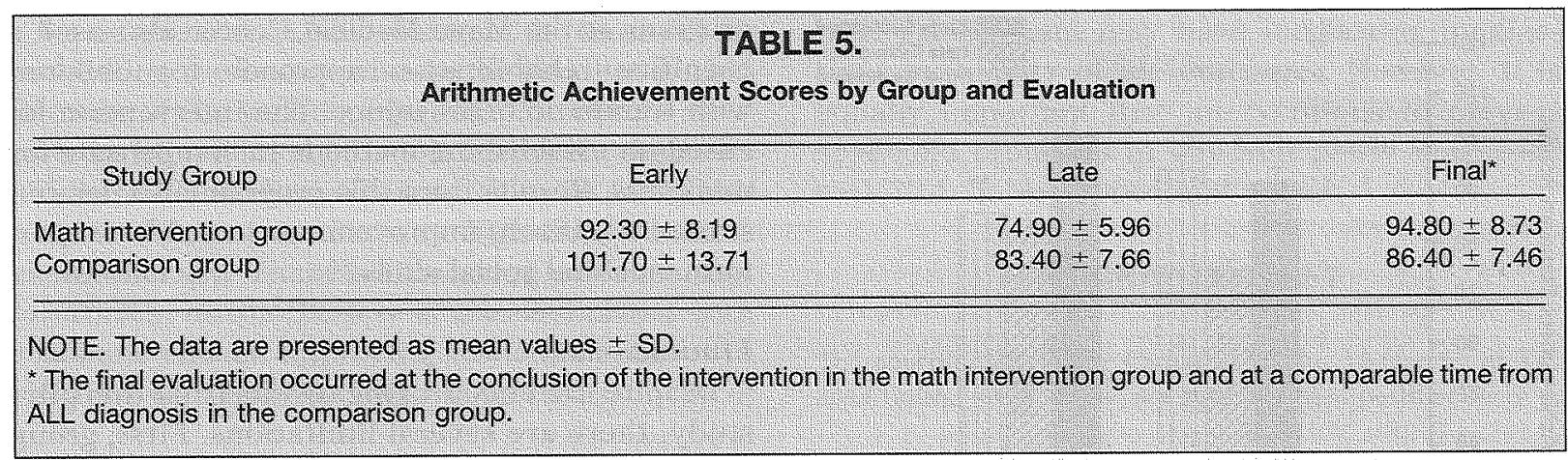

Academic arithmetic abilities were assessed in study 1 at the early and late evaluations. Academic arithmetic abilities were measured by standard scores from the WRAT-R. ${ }^{44}$ The WoodcockJohnson revised achievement battery (WJ-R) ${ }^{51}$ was used to assess math abilities at the beginning and end of the intervention. Alternative forms (A and $B$ ) were used to minimize practice effects. The WJ-R is standardized with normative data for individuals aged 5 years and older. Subtests that yield broad cluster scores in mathematics were used. Test-retest reliability coefficients of the individual subtests are 0.90 to 0.93 .51 Math abilities were assessed by the WJ-R in the no-intervention comparison group at a time consistent with the postintervention final evaluation ( 78 to 84 months after ALL diagnosis).

Data analysis. Descriptive statistics were computed on demographic variables. Fourteen of the 15 subjects completed the early, late, and final evaluations of arithmetic abilities. Therefore, data from these evaluations was used for statistical analyses. Student's $t$-test was used to compare group differences on age at ALL diagnosis and decline in academic arithmetic abilities from the early to late evaluation. One-way ANOVA followed by Student-Newman-Keuls multiple comparison test was used to compare mean academic arithmetic scores at the early, late, and final evaluations in the math intervention and the comparison groups. Level of significance was set at $\alpha=.05$ for all statistical procedures.

\section{Results}

The math intervention group included five males and three females. The mean age at the time of ALL diagnosis was $60.70 \pm 31.77$ months. There were five males and two females in the comparison group; the mean age at the time of ALL diagnosis was $66.10 \pm 5.95$ months. The mean age at the time of ALL diagnosis was not significantly different between the two groups $(t=$ 0.27 ; $\mathrm{df}=12, P=.79$ ).

Mean academic arithmetic scores at the early evaluation were lower in the math intervention group (mean, $92.3 \pm 8.19$ ) than in the comparison group (mean, $101.7 \pm 13.71$ ), but the difference was not statistically significant $(t=1.65, \mathrm{df}=13$, $P=.12$ ). Mean scores at the late evaluation declined $17.4 \pm 5.95$ standard score points in the math intervention group and $17.7 \pm 11.51$ in the comparison group. The mean decline was not significantly different between the two groups $(t=$ $-0.07, \mathrm{df}=13, P=.94)$. Mean arithmetic achievement scores at the early, late and final evaluations for each group are presented in $\mathrm{Ta}$ ble 5 .

All subjects who received the math intervention demonstrated an improvement in arithmetic abilities from the late to the final evaluation (mean, $19.90 \pm 4.09$ ). Results from the one-way ANOVA were statistically significant $(\mathrm{F}=15.7, \mathrm{df}=2, P<$ .0001 ). Post hoc analyses indicated significant differences between the early and late evaluations and between the late and final evaluations. There was no significant difference between the early and final evaluation.

Overall, a comparable increase in arithmetic abilities was not observed in the comparison group; however, two subjects did have improved arithmetic abilities at the final evaluation. Academic arithmetic scores remained stable $(n=2)$ or declined $(n=3)$ in the remaining subjects. A significant effect was also found in the comparison group $(\mathrm{F}=6.68, \mathrm{df}=2, P=.006)$; however, the differences occurred between the early and late evaluations and between the early and final evaluations. Figure 1 illustrates significant pairwise comparisons in the intervention and comparison groups. 


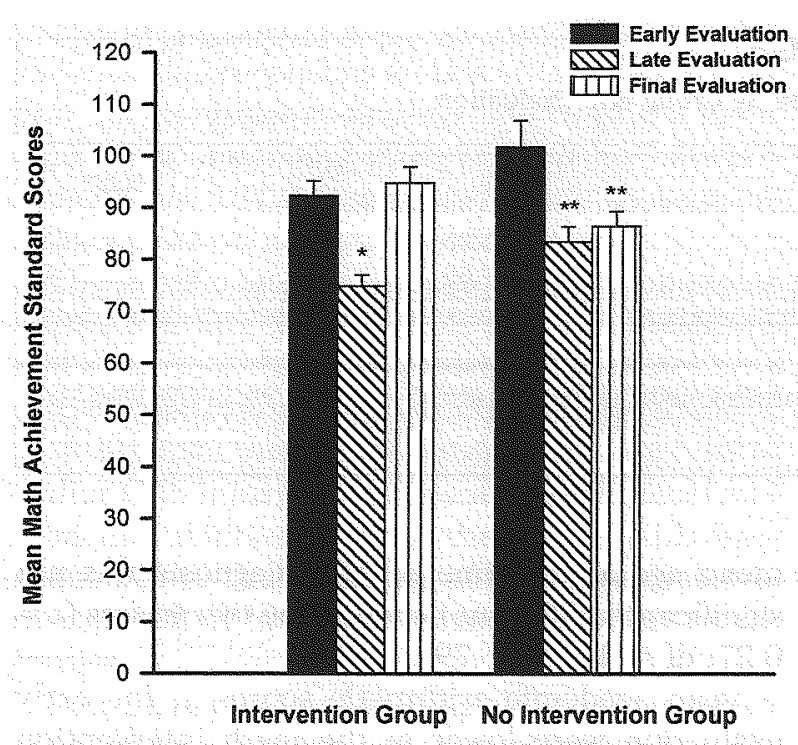

FIGURE 1. Mean math achievement scores by group and evaluation. *Intervention group late evaluation mean score significantly lower than early and final evaluation mean scores. **No intervention group late and final mean scores significantly lower than early evaluation mean score.

Academic arithmetic abilities declined approxinately 17 points in both groups over approximately 46 months. The WRAT-R and WJ-R standard scores are based on a mean of 100 and a standard deviation of 15 ; therefore, the average decline in arithmetic abilities in both groups represents a large effect size. Arithmetic abilities increased a mean of $19.90 \pm 4.09$ standard score points from the late to the final evaluation in the math intervention group. Using a conservative estimate, the math intervention had a moderate to large effect on academic arithmetic abilities.

\section{Discussion}

To our knowledge this is the first report of an intervention designed to improve arithmetic abilities in children with ALL who received GNS treatment. Preliminary findings from the eight subjects suggest that the skill-based tutorial approach was an effective method for teaching math concepts and improving math abilities. Remedial cognitive interventions have been effective in children who sustained a traumatic brain injury. Boyer and Edwards ${ }^{52}$ documented measurable improvement in education and cognitive function for up to 3 years following a rehabilitation intervention involving 220 children and adolescents with traumatic brain injury. The brain injury outpatient program at the Mayo Medical Center also used a cognitive rehabilitation approach for children with traumatic brain injury. The intervention focused on establishing methods to compensate for weakness. Results from this program also demonstrated significant improvements in academic performance and social skills. ${ }^{53}$

\section{Limitations}

Despite the encouraging results from this pilot study, there are several limitations. First, group assignment was not random. Therefore, selection bias must be considered. It is also possible that some children were receiving other forms of educational assistance. Another limitation is the use of two standardized measures of academic arithmetic abilities. The WRAT-R was used in study 1 while the WJ-R was used in the pilot intervention. The rationale for using the WJ-R was the availability of alternate forms to minimize practice effects in the math intervention group. While these two measures of academic arithmetic are correlated, scores on the final evaluation of academic arithmetic compared with those from early and late evaluations may be influenced to some extent by the use of different instruments.

\section{Conclusions}

The findings from this pilot study are encouraging and suggest that the math intervention improved arithmetic abilities in children with declines in this area of academic performance as a result of CNS treatment for ALL. The initial estimate suggests that the intervention effect size was at least moderate, and therefore has the potential to have a clinically significant impact on academic late effects. Additional studies, however, are needed to substantiate the results reported here. The intervention needs to be tested in a randomized study involving a larger sample of children to minimize threats to internal validity, such as selection bias and history. Other factors that could influence academic performance, such as missed school, and parents' efforts to obtain additional assistance, such as special education, also need to be considered in future studies. Finally, it is important to determine whether skill-based tutorial interventions have a sustained effeet on academic outcomes or if additional "booster" interventions are needed as the child progresses to more complex academic and cognitive tasks. 
In conclusion, GNS treatment is essential to preventing meningeal disease in childhood ALL. Because of the well-documented late effects associated with radiation, ${ }^{5-19}$ most treatment regimens use intrathecal chemotherapy alone or in combination with intermediate- to high-dose systemic chemotherapy. There is increasing evidence that intrathecal chemotherapy is also associated with academic effects. Improving academic abilities in children who have received GNS treatment is of high priority and may have longlasting implications on quality of life outcomes. However, it is equally important that interventions to prevent academic problems in children newly diagnosed with ALL be developed and tested.

\section{ACKNOWLEDGMENT}

The contributions of Dr Catherine Locke, University of Arizona College of Medicine, are acknowledged posthumously

\section{ReFERENCES}

1. Greenlee, RT, Murray T, Bolden S, et al: Cancer statistics 2000. CA Cancer J Clin 50:7-33, 2000

2. Pui CH: Childhood leukemias.. N Engl J Med 332:1618 1630,1995

3. Evans AE, Gilbert $S E$, Zandstra R: The increasing incidence of central nervous system leukemia in children. Gancer 26:404-409, 1970

4. Balis FM, Poplack DG: Central nervous system pharmacology of antileukemic drugs. Am J Pediatr Hematol Oncol $11: 74-86,1989$

5. Anderson V, Smibert E, Ekert H, et al: Intellectual, educational, and behavioural sequelae after cranial irradiation and chemotherapy. Arch Dis Child 70:476-485, 1994

6. Butler RW, Hill JM, Steinherz PG, et al: Neuropsychologic effects of cranial irradiation, intrathecal methotrexate, and systemic methotrexate in childhood cancer. J Clin Oncol 12: 2621-2629, 1994

7. Christie D, Leiper AD, Chessells JM, et al: Intellectual performance after presymptomatic cranial radiotherapy for leukaemia. Effects of age and sex. Arch Dis Child 73:136-140, 1995

8. Cousens $\mathrm{P}$, Waters B, Said J, et al: Cognitive effects of cranial irradiation in leukaemia: $A$ survey and meta-analysis. J Child Psychol Psychiatry 29:839-852, 1988

9. Dowell RE, Copeland DR, Francis DJ, et al: Absence of synergistic effects of CNS treatments on neuropsychologic test performance among children. J Clin Oncol 9:1029-1036, 1991

10. Giralt $\mathrm{J}$, Ortega $\mathrm{JJ}$, Olive $\mathrm{T}$, et al: Long-term neuropsychologic sequelae of childhood leukemia: Comparison of two GNS prophylactic regimens. Int J Radiat Oncol Biol Phys $24: 49-53,1992$

11. Halberg FE, Kramer JH, Moore IM, et al: Prophylactic cranial irradiation dose effects of late cognitive function in children treated for acute lymphoblastio leukemia. Int J Radiat Oncol Biol Phys 22:13-16, 1991.

12. Hongwei $Q$, Jianjun $F$, Yonghong $Z$, et al: Intelligence function in children with acute lymphoblastic leukemia after treatment. Chin Med Sci J 8:91-94, 1992

13. Jankovic M, Brouwers P, Valsecchi MG, et al Association of 1800 cGy cranial irradiation with intellectual function in children with acute lymphoblastic leukaemia. Lancet 344 . 224-227, 1994

14. Kato M, Azuma E, Ido M, et al: Ten-year survey of the intellectual deficits in children with actite Iymphoblastic leukemia receiving chemoimmunotherapy. Med Pediatr Oncol $2.435-440,1993$

15. Kleinman SN, Waber DP: Prose memory strategies of children treated for leukemia: A story grammar analysis of the Anna Thompson passage: Neuropsychology 8:464-470, 1994

16. MacLean WE, Noll RB, Stehbens JA, et al: Neuropsychological effects of cranial irradiation in young children with acute lymphoblastic leukemia 9 months after diagnosis. Arch Neurol 52:156-160,1995

17. Rodgers J, Britton PG, Morris RG, et al: Memory after treatment for acute lymphoblastic leukaemia. Arch Dis Ghild 67:266-268, 1992

18. Smibert E, Anderson V, Godber T, et al: Risk factors for intellectual and educational sequelae of cranial irradiation in childhood acute lymphoblastic leukemia. Br J Cancer 73:825830,1996

19. Waber DP, Bernstein JH, Kammerer BL, et al: Neuropsychological diagnostic profiles of children who received CNS treatment for acute lymphoblastic leukemia. The systemic approach to assessment. Dev Neuropsychol 8:1-28, 1992

20. Waber DP, Tarbell NJ, Kahn CM, et al: The relationship of sex and treatment modality to neuropsychological outcome in childhood acute lymphoblastic leukemia. J Cilin Oncol 10 : $810-817,1992$

21. Williams KS, Ochs J; Williams MJ, et al: Parental report of everyday cognitive abilities among children treated for acute lymphoblastic letkemia. J Pediatr Psychol 16:13-26, 1991

22. Brown RR, Sawyer MB, Antoniou $G$, et al: A 3 year follow-up of the intellectual and academic functioning of children receiving central nervous system prophylactic chemotherapy for leukemia. J Dev Behav Pediatr 17:392-398, 1996

23. Copeland DR, Moore BD, Francis DJ, et al: Neuropsy chologic effects of chemotherapy on children with cancer-A longitudinal study. J Clin Oncol 14:2826-2835, 1996

24. Mulhern RK, Fairclough D, Ochs J: A prospective comparison of neuropsychologic performance of children surviving leukemia who received $18-\mathrm{Gy}, 24-\mathrm{Gy}$, or no cranial irradiation. J. Clin Oncol 9:1348-1356, 1991

25. Ochs J, Mulhern R, Fairclough D, et al Comparison of neuropsychologic functioning and clinical indicators of neurotoxicity in long-term survivors of childhood leukemia given cranial radiation of parenteral methotrexate. A prospective study. J Clin Oncol 9:145-151, 1991

26. Espy KA, Moore IM, Kaufmann PM, et al Chemotherapeutic CNS prophylaxis and neuropsychologic change in children with acute lymphoblastic leukemia: A prospective study. $\mathrm{J}$ Pediatr Psychol (in press)

27. Asato $\mathrm{R}$, Akiyama $\mathrm{Y}$; Ito $\mathrm{M}$, et al: Nuclear magnetio resonance abnormalities of the cerebral white matter in children with acute lymphoblastic leukemia and malignant lym- 
phoma during and after central nervous system prophylactic treatment with intrathecal methotrexate. Gancer 70:19972004, 1992

28. Harila-Saari AH, Ahonen KA, Vainionpää LK, et al: Brain perfusion after treatment of childhood acute lymphoblastic leukemia. J Nucl Med 38:82-88, 1997

29. Hertzberg $H$, Huk WJ, Ueberall MA, et al: CNS late effects after all therapy in childhood part I: Neuroradiological findings in long-term survivors of childhood ALL-An evaluation of the interferences between morphology and neuropsychological performance. Med Pediatr Oncol 28:387-400, 1997

30. Kingma A, Mooyaart EL, Kamps WA, et al: Magnetic resonance imaging of the brain and neuropsychological evaluation in children treated for acute lymphoblastic leukemia at a young age. Am J Pediatr Hematol Oncol 15:231-238, 1993

31. Moore BD III, Copeland DR, Ried H, et al: Neurophysiological basis of cognitive deficits in long-term survivors of childhood cancer. Arch Neurol 49:809-817, 1992

32. Phillips PC, Moeller JR, Sidtis JJ, et al: Abnormal cerebral glucose metabolism in long-term survivors of childhood acute lymphocytic leukemia. Ann Neurol 29:263-271, 1991

33. Prassopoulos P, Cavouras D, Golfinopoulos S, et al: Quantitative assessment of cerebral atrophy during and after treatment in children with acute lymphoblastic leukemia. Invest Radiol 31:749-754, 1996

34. Sato Y, Miyao M, Muchi H, et al: P300 as indicator of effects of prophylactic cranial radiation. Pediatr Neurol 8:130132,1992

35. Seidel H, Nygaard R, Moe PJ: Magnetic resonance imaging and neurological evaluation after treatment with high-dose methotrexate for acute lymphocytic leukaemia in young children. Acta Paediatr 85:450-453, 1996

36. Ueberall MA, Wenzel D, Hertzberg $\mathrm{H}$, et al: CNS late effects after all therapy in childhood. Part II: Conventional EEG recordings in asymptomatic long-term survivors of childhood ALL-An evaluation of the interferences between neurophysiology, neurology, psychology, and CNS morphology. Med Pediatr Oncol 29:121-131, 1997

37. Dugan LL, Choi DW: Excitotoxicity, free radicals, and cell membrane changes. Ann Neurol 35:S17-S21, 1994

38. Mollova NN, Moore IM, Hutter J, et al: Fast atom bombardment mass spectrometry of phospholipids in human cerebrospinal fluid. J Mass Spectrom 30:1405-1420, 1995

39. Illingworth $\mathrm{DR}$, Glover $\mathrm{J}$ : The composition of lipids in cerebrospinal fluid of children and adults. J Neurochem 18: 769-776, 1971

40. Folch J, Lees M, Stanley GHS: A simple method for the isolation and purification of total lipids from animal tissues. J Biol Chem 226:497-509, 1957
41. Ghen SF, Chan PH: One-step separation of free fatty acids and phospholipids in brain tissue extracts by high-performance liquid chromatography. J Chromatogr 344:297-303, 1985

42. Wechsler D: Manual for the Wechsler Intelligence Scale for Children. New York, NY, Revised Psychological Corporation, 1974

43. McCarthy D: Manual: McCarthy Scales of Children's Abilities. New York, NY, Harcourt Brace Jovanovich Psychological Corporation, 1972

44. Jastak S, Wilkinson G: The Wide Range Achievement Test. Revised Administration Manual. Wilmington, DE, Jastak Associate, 1984

45. Chan PH, Longar S, Fishman RA: Phospholipid degradation and edema development in cold-injured rat brain. Brain Res 277:329-337, 1983

46. Chan PH, Fishman RA, Longar S, et al: Cellular and molecular effects of polyunsaturated fatty acids in brain ischemia and injury. Prog Brain Res 63:227-235, 1985

47. Mahoney DH, Nitschke R, Lauer S, et al: Neurotoxicity in children with acute lymphoblastic leukemia receiving intense methotrexate schedules: A Pediatric Oncology Group study. Proc Am Soc Clin Oncol 15:366A, 1996 (abstr)

48. Mahoney DH, Shuster J, Nitschke R, et al: Intermediatedose intravenous methotrexate with intravenous mercaptopurine is superior to repetitive low-dose oral methotrexate with intravenous mercaptopurine for children with lower-risk Blineage acute lymphoblastic leukemia: A Pediatric Oncology Group phase III trial. J Clin Oncol 16:246-254, 1998

49. Surtees R, Clelland J, Hann I: Demyelination and singlecarbon transfer pathway metabolites during the treatment of acute lymphoblastic leukemia: CSF studies. J Clin Oncol 16: 1505-1511, 1998

50. Quinn CT, Griener JC, Bottiglieri T, et al: Evaluation of homocysteine and excitory amino acid neurotransmitters in the CSF of children who receive methotrexate for the treatment of cancer. J Clin Oncol 15:2800-2806, 1997

51. Woodcock RW, Mather N: Woodcock-Johnson Tests of Achievement-Revised: Examiner's Manual. Allen, TX, DLM Teaching Resources, 1990

52. Boyer MG, Edwards P: Outcome 1 to 3 years after severe traumatic brain injury in children and adolescents. Injury 22:315-320, 1991

53. Smigielski JS, Malee JF, Thompson JM, et al: Subspecialty clinics: Physical medicine and rehabilitation. Mayo Medical Center Brain Injury Outpatient Program: Treatment procedures and early outcome data. Mayo Clin Proc 67:767-774, 1992

Ida M. Moore, DNS, FAAN: University of Arizona College of Nursing, Tucson; Kimberly Andrews Espy, PhD: Southern Illinois University School of Medicine, Carbondale; Paul Kaufmann PhD; Southern Illinois University School of Medicine, Carbondale; Joel Kramer, PhD: University of California San Francisco Medical Center; Kris Kaemingk, PhD: University of Arizona College of Medicine, Tucson; Petra Miketova, PhD: University of Arizona College of Nursing, Tucson; Nevena Mollova, PhD: University of Arizona College of Nursing, Tucson; Marta Kaspar, MS: University of Arizona College of Nursing, Tucson; Alice Pasvogel, PhD: University of Arizona College of Nursing, Tucson; Karl Schram, PhD: University of Arizono College of Pharmacy, Tucson; William Wara, PhD: University of California San Francisco Medical Center; John Hutter, MD: University of Arizona College of Medicine, Tucson; and Kate Matthay, MD: University of California San Francisco Medical Center. 